\title{
Measurement and thermal modeling of sapphire substrate temperature at III-Nitride MOVPE conditions
}

\author{
J. Randall Creighton, Michael E. Coltrin* and Jeffrey J. Figiel* \\ JR Creighton Consulting \\ 4005 Embudito Dr. NE \\ Albuquerque, NM 87111 \\ and \\ *Sandia National Laboratories, \\ P.O. Box 5800, MS-1086 \\ Albuquerque, New Mexico, 87185 \\ Corresponding author E-mail: randy.creighton.sandia@gmail.com
}

Ph: 505-715-0657, FAX: 505-844-3211 


\section{Abstract}

Growth rates and alloy composition of AlGaN grown by MOVPE is often very temperature dependent due to the presence of gas-phase parasitic chemical processes. These processes make wafer temperature measurement highly important, but in fact such measurements are very difficult because of substrate transparency in the near-IR $(\sim 900 \mathrm{~nm})$ where conventional pyrometers detect radiation. The transparency problem can be solved by using a mid-IR pyrometer operating at a wavelength $(\sim 7500 \mathrm{~nm})$ where sapphire is opaque. We employ a mid-IR pyrometer to measure the sapphire wafer temperature and simultaneously a near-IR pyrometer to measure wafer pocket temperature, while varying reactor pressure in both a $\mathrm{N}_{2}$ and $\mathrm{H}_{2}$ ambient. Near $1300^{\circ} \mathrm{C}$, as the reactor pressure is lowered from 300 Torr to 10 Torr the wafer temperature drops dramatically, and the $\Delta \mathrm{T}$ between the pocket and wafer increases from $\sim 20^{\circ} \mathrm{C}$ to $\sim 250^{\circ} \mathrm{C}$. Without the mid-IR pyrometer the large wafer temperature change with pressure would not have been noted. In order to explain this behavior we have developed a quasi-2D thermal model that includes a proper accounting of the pressure-dependent thermal contact resistance, and also accounts for sapphire optical transmission. The model and experimental results demonstrate that at most growth conditions the majority of the heat is transported from the wafer pocket to the wafer via gas conduction, in the free molecular flow limit. In this limit gas conductivity is independent of gap size but first order in pressure, and can quantitatively explain results from 20-300 Torr. Further analysis yields a measure of the thermal accommodation coefficients; $\alpha(\mathrm{H} 2)=0.23, \alpha(\mathrm{N} 2)=0.50$, which are in the range typically measured.

Keywords: A3. Metalorganic vapor phase epitaxy; A3. Metalorganic chemical vapor deposition; A3. Organometallic vapor phase epitaxy; A1. Heat transfer; B1. Nitrides 


\section{Introduction}

Parasitic chemical processes during III-Nitride MOVPE often create a strongly temperature dependent film deposition rate [1-15], which makes temperature measurement critical for control of film thickness and alloy composition. Unfortunately, the visible and near-IR transparency of the substrates typically used (sapphire, GaN, AlN, SiC) prevents the use of conventional pyrometry to directly measure substrate temperatures. These approaches actually measure the wafer carrier, or wafer pocket temperature, and are thus only an indirect proxy of the true wafer temperature. Alternative pyrometric methods based on GaN opacity at $\sim 400 \mathrm{~nm}$ and sapphire opacity in the mid-IR $(\sim 7500 \mathrm{~nm})$ have the ability to directly measure the true wafer temperature [16-18], but widespread adoption of these tools has not yet occurred. We also note that the near-UV or $\sim 400 \mathrm{~nm}$ pyrometer is not viable unless an absorbing layer of $\mathrm{GaN}$ is present, which is not typically the case when growing AlN or high Al-content AlGaN layers for UV-emitters or power electronic applications, leaving the mid-IR pyrometer as the only option.

Despite their disadvantages indirect temperature measurement methods are still most often employed because of their ease of use. One widely used indirect method is near-IR pyrometry with some type of emissivity correction added to account for changes in film transmission (via a reflection measurement) $[19,20]$. Unfortunately the temperature offset between the wafer and wafer pocket is dependent on many MOVPE process variables (pressure, flow rate, gas composition), thus making the indirect methods less reliable. One noticeable example occurs during the MOVPE of AlN (and high Al content $\mathrm{AlGaN}$ ), which is often grown at lower total pressures (e.g. $<100$ Torr) in order to minimize gas-phase parasitic chemical reactions. The effective thermal contact resistance between the wafer and substrate becomes very pressure dependent at these conditions, and temperature differences between the carrier and wafer, $\Delta \mathrm{T}=\left(\mathrm{T}_{\text {carrier }}-\mathrm{T}_{\text {wafer }}\right)$, can be greater than $100^{\circ} \mathrm{C}$ [16]. If only an indirect method is used this wafer temperature change would not be seen, and any change in growth rate, composition, etc. might be erroneously ascribed to a pressure change alone. 
To study this effect in more detail we have used a mid-IR pyrometer to directly measure the sapphire wafer temperature, near $1300^{\circ} \mathrm{C}$, in a $\mathrm{N}_{2}$ and $\mathrm{H}_{2}$ ambient, at pressures in the $10-300$ Torr range. The susceptor temperature was also measured with a conventional near-IR pyrometer, to yield accurate $\Delta \mathrm{T}$ values. In order to explain the experimental observations we have developed a quasi-2D thermal model that includes a proper accounting of the pressure-dependent thermal contact resistance. The model also includes radiative heat exchange using the gray body approximation, with an extension to include the effect of sapphire transmission. One future goal of this work would be to generate a model with sufficient accuracy to serve as a supplement to the indirect methods, when no absolute temperature measurement method is available. 


\section{Experimental Procedure and Results}

The experiments were performed in a custom single 2-inch wafer (2") reactor using a design provided by North Carolina State University [21-23]. The general reactor design is similar to our earlier rotating disk reactors (RDR's), with vertical gas injection from the top impinging on a wafer perpendicular to the gas stream [1,24-26], although details of the gas injection and wafer loading are now somewhat different. The addition of a $30 \mathrm{~kW}$ RF generator and low emissivity TaC-coated graphite susceptor enables the system to reach temperatures of $\sim 1600^{\circ} \mathrm{C}$, with our aim to explore high temperature AlN and AlGaN MOVPE. In this study rotation rates were kept at or below $\sim 200 \mathrm{rpm}$, which is too low to exert any significant influence on the gas flow, but high enough to help smooth out any temperature irregularities due to RF coupling variations.

Temperatures were measured with two types of pyrometers. A standard near-IR ( 900 nm) pyrometer (IRCON 5R-1810100, operated in single color mode) was used to measure the TaC-coated susceptor temperature, normally looking at the center of the wafer pocket. Even with a sapphire wafer in place, this pyrometer only observes the radiation emitted from the susceptor, as the wafer remains transparent (no significant absorption) in the near-IR even at very high temperatures [27]. After accounting for wafer transmission losses solely due to reflection, the susceptor temperature in the wafer pocket can be accurately measured. A second, mid-IR $(\sim 7500 \mathrm{~nm})$ pyrometer was used to measure the sapphire wafer temperature, as has been described in more detail previously [17]. At this wavelength sapphire is opaque, so it is possible to measure temperature radiometrically. Using these two pyrometers allows simultaneous measurement of both the wafer pocket and sapphire wafer temperatures.

Typically there will be a temperature difference (offset) between the pocket and wafer, and this offset is potentially dependent upon reactor conditions (pressure, gas-composition, flow rate, etc.). Changes in reactor pressure are particularly significant, as shown in Fig. 1; one set of curves is for pure $\mathrm{N}_{2}$ and the other pure $\mathrm{H}_{2}$. At higher pressures ( $>100$ Torr) the offset is typically $10-30^{\circ} \mathrm{C}$, and nearly 
independent of pressure, but as the pressure is lowered below 100 Torr the sapphire temperature begins to drop precipitously, while the wafer pocket temperature actually increases slightly (RF power held constant). At 10 Torr the offsets are $253^{\circ} \mathrm{C}\left(\mathrm{H}_{2}\right)$ and $294^{\circ} \mathrm{C}\left(\mathrm{N}_{2}\right)$. Without the mid-IR pyrometer these large temperature changes in the 10-100 Torr pressure range would not be apparent, and therefore variations in $\mathrm{AlN}$ or $\mathrm{AlGaN}$ growth rate and/or material properties (e.g. alloy composition, impurity concentrations, etc.) could be erroneously assigned to pressure changes alone. A similar trend with pressure was observed previously using a $400 \mathrm{~nm}$ pyrometer for GaN-coated sapphire, but not quantitatively explained [16].

\section{Thermal Model Description and Results}

The behavior of the temperature offset seen in Fig. 1 is thought to arise from the thermal contact resistance (or a portion of it) being pressure dependent, an effect that has been studied in many physical systems $[28,29]$. The physical basis of this observation is that when the mean free path $(\Lambda)$ becomes comparable to or larger than the distance $(\mathrm{d})$ between two surfaces $(\mathrm{Kn}=\Lambda / \mathrm{d}>1)$, heat exchange via gas conductivity no longer follows the behavior in the continuum limit. As a point of reference $\Lambda\left(\mathrm{N}_{2}\right)$ is $\sim 20$ microns at $1000^{\circ} \mathrm{C}$ and 10 Torr, and the gap size is typically a few microns (in the absence of significant wafer bow). In the continuum limit the heat flux $(\mathbf{q})$ is nearly independent of pressure and inversely proportional to distance. In contrast, in the free molecular flow regime $\mathbf{q}$ molf is first order in pressure and independent of distance, obeying equation 3.1 [ref 30 eqn. 1.136].

$$
q_{m o l f}=\frac{\alpha}{8}\left(\frac{\gamma+1}{\gamma-1}\right) \frac{P \cdot v}{T} \Delta T
$$

Here $\alpha$ is an effective thermal accommodation coefficient (vide infra), $\gamma=\mathrm{Cp} / \mathrm{Cv}$ (heat capacity at constant pressure/heat capacity at constant volume; typically $\approx 1.4$ for diatomic gases), and $v=$ average molecular velocity. 
This heat flow mechanism is in fact very important for most cold-wall MOVPE environments, and as we will see is often the dominant path by which heat flows from the susceptor (or any wafer carrier) to the wafer. In addition to $\boldsymbol{q}_{\boldsymbol{m} \text { olf }}$, there are potentially two parallel pathways for heat flow; radiation exchange $\left(\boldsymbol{q}_{\text {Irad }}\right)$ and conduction through solid points of contact. We find no evidence the latter plays a role so it is not included in the following discussion. As a point of reference for the remainder of the discussion, refer to the equivalent thermal circuit diagram; Fig. 2. The two terms just described; $\boldsymbol{q}_{\text {molf }}$ and $\boldsymbol{q}_{1 \text { rad }}$ represent the heat flux flowing through $\mathbf{R}_{\text {molf }}$ and $\mathbf{R}_{1 \text { rad }}$, respectively, and represent heat flow from the susceptor wafer pocket (node 1 at temperature $T_{1}$ ) to the sapphire wafer (node 2 at temperature $\mathrm{T}_{2}$ ). The third node is the reactor wall, and heat flowing from the wafer to the reactor wall occurs via emitted radiation $\left(\boldsymbol{q}_{2 \text { rad }}\right)$ and gas convection/conduction (combined into one term; $\boldsymbol{q}_{\text {gas }}$ ).

In this study we have made a simplifying approximation in regard to the wafer itself; we are ignoring the temperature gradient through the wafer, and treating it as if it had zero thickness. At the power densities of interest $\left(\sim 10 \mathrm{~W} / \mathrm{cm}^{2}\right)$ the temperature gradient across a standard 430 micron thick sapphire wafer is about $5^{\circ} \mathrm{C}$, which is relatively small compared to the temperature gradients of interest (10's to 100 's of degrees). Furthermore, this small gradient does not change appreciably when we are examining the effect of pressure at constant RF power, so it only introduces a small offset.

One property of the sapphire that requires special note is that it is transparent from the UV to the mid-IR, even at very high temperatures $\left(>1200^{\circ} \mathrm{C}\right)[27,31]$. The sapphire absorption edge in the mid-IR blue-shifts with increasing temperature; going from $\sim 5000 \mathrm{~nm}$ at room temperature to $4000 \mathrm{~nm}$ at $1400^{\circ} \mathrm{C}$. For a black-body (or gray body) at temperatures above $\sim 700^{\circ} \mathrm{C}$ more than $50 \%$ of the total radiative power is in the band below $4000 \mathrm{~nm}$ [32]. Therefore the effect of sapphire transmission on radiative coupling is very significant; a large fraction of radiation from the susceptor simply passes through the sapphire wafer without absorption. For simplicity we will model this effect using the gray body approximation, extended to include sapphire transmission. 
An analysis of the radiative fluxes between two surfaces, with one material being semitransparent $(0<\tau<1)$ is shown in Fig. 3; using the gray-body approximation framework [32]. The surfaces are assumed to be semi-infinite, and multiple reflections/transmissions are summed using the $1+x+x^{2}+$ $x^{3}+\cdots=\frac{1}{1-x}$ identity. Six terms are derived, corresponding to the fluxes emitted $\left(\boldsymbol{q}_{\text {emit }}\right)$, reflected $\left(\boldsymbol{q}_{\text {refl }}\right)$ and transmitted $\left(\boldsymbol{q}_{\text {trans }}\right)$ for each surface. The four fluxes for emission and reflection are used to define the net radiative flux $\left(\boldsymbol{q}_{1} \mathbf{r a d}\right)$ flowing from 1 to 2 , which is given by Eqn. $3.2(\sigma=$ StefanBoltzmann constant, $\varepsilon_{1}=$ emissivity of $\mathbf{1}$, etc.). The two transmitted fluxes are summed to define $\boldsymbol{q}_{\text {trans }}$, as shown in Eqn. 3.3. Note when $\tau_{2}=0$ eqn. 3.2 reduces to the standard expression for two nontransparent surfaces [32], and eqn. $3.3=0$. The remaining radiation flux is that emitted from 2 towards the reactor wall $\left(\boldsymbol{q}_{2 r a d}\right)$, and is given in Eqn. 3.4. Finally, the expression for $\boldsymbol{q}_{\text {gas }}$ is given by eqn. 3.5, where $\mathbf{R}_{\text {gas }}$ may have contributions from both convective and conductive heat transfer.

$$
\begin{aligned}
& q_{1 \text { rad }}=q_{1 \text { emit }}-q_{1 \text { refl }}-q_{2 \text { emit }}+q_{1 \text { refl }}=\sigma \frac{\varepsilon_{1}\left(\varepsilon_{2}+\tau_{2}\right) T_{1}^{4}-\varepsilon_{1} \varepsilon_{2} T_{2}^{4}}{1-R_{1} R_{2}} \\
& q_{\text {trans }}=q_{1 \text { trans }}+q_{2 \text { trans }}=\sigma \frac{\varepsilon_{1} \tau_{2} T_{1}^{4}+\varepsilon_{2} R_{1} \tau_{2} T_{2}^{4}}{1-R_{1} R_{2}} \\
& q_{2 \text { rad }}=\sigma \varepsilon_{2}\left(T_{2}^{4}-T_{w}^{4}\right) \\
& q_{g a s}=\frac{T_{2}-T_{w}}{R_{g a s}}
\end{aligned}
$$

These five heat flux terms (Eqns. 3.1-3.5) are then used in two equations defining the total heat flux flowing through the sapphire wafer $\left(\boldsymbol{\rho}_{\text {waf }}\right)$. Heat flow between the susceptor and wafer is given by Eqn. 3.6, and heat flow between the wafer and reactor wall is given by Eqn. 3.7.

$$
\begin{aligned}
& \rho_{\text {waf }}=q_{1 \text { rad }}+q_{\text {molf }} \\
& \rho_{\text {waf }}=q_{2 \text { rad }}+q_{\text {trans }}+q_{\text {gas }}
\end{aligned}
$$


At this point, if $\boldsymbol{\rho}_{\text {waf }}$ is a constant and all other physical properties are defined, we can solve the non-linear Eqns. 3.6 and 3.7 to yield $\mathrm{T}_{1}$ and $\mathrm{T}_{2}$. In fact, using this boundary condition $\left(\boldsymbol{\rho}_{\mathrm{waf}}=\right.$ constant) yields unsatisfactory results; as $\mathbf{R}_{\text {molf }}$ is increased (equivalent to lowering the pressure) $\mathrm{T}_{1}$ increases much more than is observed experimentally, while $\mathrm{T}_{2}$ only drops slightly. If we use a constant $\mathrm{T}_{1}$ boundary condition we can recover a large drop in $\mathrm{T}_{2}$ as the pressure is lowered (as observed experimentally), but we lose the small increase in $\mathrm{T}_{1}$.

We were able to make a substantial improvement in behavior by making the model "quasi-2D". The susceptor is divided into two sections; one section is the wafer pocket with the wafer in place, while the second larger section is simply the remainder of the susceptor. The heat flux through the remainder of the susceptor ( $\boldsymbol{\rho}_{\text {ros }}$ ) is given by Eqn. 3.8. The susceptor is assumed to be isothermal, but $\boldsymbol{\rho}_{\text {waf }}$ and $\boldsymbol{\rho}_{\text {ros }}$ are not necessarily equal. In this case the total power $\left(\mathrm{P}_{\text {total }}\right)$ is given by Eqn. 3.9, and this value is held fixed as a boundary condition. This segmentation allows some power redistribution to occur in response to changes in reactor conditions, and greatly improves the behavior of the model.

$$
\begin{aligned}
& \rho_{\text {ros }}=\sigma \frac{\varepsilon_{1} \varepsilon_{w}\left(T_{1}^{4}-T_{w}^{4}\right)}{\varepsilon_{1}+\varepsilon_{w}-\varepsilon_{1} \varepsilon_{w}}+\frac{T_{1}-T_{w a l}}{R_{g a s}} \\
& P_{\text {total }}=A_{\text {waf }} \cdot \rho_{\text {waf }}+A_{\text {ros }} \cdot \rho_{\text {ros }} \\
& A_{\text {waf }}=\text { wafer area, } A_{\text {ros }}=\text { remainder of susceptor area }
\end{aligned}
$$

The wafer area is known $\left(20.3 \mathrm{~cm}^{2}\right)$, and we compute the area the rest of the susceptor using the known dimensions of the main cylindrical section $(7.62 \mathrm{~cm}$ diameter $X 3.18 \mathrm{~cm}$ height), but not including the $1.27 \mathrm{~cm}$ diameter supporting shaft. Since a portion of the support shaft does get hot this approximation tends to underestimate the surface area by a small amount. Defining $\mathrm{P}_{\text {total }}$ is not without some difficulty; we know the total RF power generated but not all of it is absorbed by the susceptor. A series of baseline tests were done in the power range of interest without the susceptor in place, and it 
was found that $\sim 50 \pm 5 \%$ of the RF power $\left(\mathrm{P}_{\mathrm{RF}}\right)$ is in fact dissipated elsewhere. Therefore the power absorbed is given by $\mathrm{P}_{\text {total }}=0.5 \mathrm{X} \mathrm{P}_{\mathrm{RF}}$.

To solve the model equations we also need values for four optical properties $\left(\varepsilon_{1}, \varepsilon_{2}, \tau_{2}\right.$, and $\left.\varepsilon_{\mathrm{w}}\right)$, along with estimates of $\mathbf{R}_{\text {gas }}$ and the thermal accommodation coefficient ( $\alpha$ ). At high temperatures $\left(1100-2000^{\circ} \mathrm{C}\right)$ the total hemispherical emissivity of $\mathrm{TaC}\left(\varepsilon_{1}\right)$ is in the range of $0.4-0.5$ [ref 31 , data table 299], and we chose an average value of 0.45. Choosing appropriate values of $\varepsilon$ and $\tau$ for sapphire is more difficult, as a great deal of the tabulated values are for polycrystalline alumina of varying purity and crystal grain size. Above $1100^{\circ} \mathrm{C}$ most values for alumina are in the 0.3-0.5 range [ref 31, figure 48A, and 59], with the expectation that high purity crystalline sapphire would be in the lower end of this range. We therefore chose $\varepsilon_{2}=0.35$. This $\varepsilon$ value, and an average 2 -surface reflectance value of 0.15 fixes a value of $\tau_{2}=0.5\left(\tau_{2}=1-\varepsilon_{2}-R_{2}\right)$. Determining an appropriate value of the wall emissivity also requires some level of approximation, given its geometry and complexity (the reactor wall is predominately fused silica; concentric tubes with water in the gap for cooling, but the gas injector is entirely stainless steel). The radiative component in Eqn. 3.8 is written to allow for some backreflection of radiation from the susceptor when $\varepsilon_{\mathrm{w}}<1$, and currently we are essentially treating $\varepsilon_{\mathrm{w}}$ as an adjustable parameter to control the magnitude of this effect. One rationale for doing this is when we use our best guesses for $\mathrm{P}_{\text {total }}$ and all the other physical properties, the computed susceptor temperature (with $\varepsilon_{\mathrm{w}}=1$ ) is typically $50-100^{\circ} \mathrm{C}$ lower than the measured value (in the $1300^{\circ} \mathrm{C}$ range). Of course it is possible we have underestimated $\mathrm{P}_{\text {total }}$, and/or overestimated $\varepsilon_{1}$, but by allowing for some degree of radiative back-reflection $\left(\varepsilon_{\mathrm{w}}<1\right)$ we can raise the calculated temperature to match the experimental measurement using $\varepsilon_{\mathrm{w}} \sim 0.8$.

To estimate the values of $\mathrm{R}_{\mathrm{gas}}$ a $2 \mathrm{D}$ model of fluid flow and heat transfer in the reactor was solved using the Ansys Fluent software [33]. Geometrical features of the reactor were used, with a fixed outerwall temperature boundary condition of $100^{\circ} \mathrm{C}$ and disk temperature of $1300^{\circ} \mathrm{C}$. The model calculates the temperature and velocity fields as gas enters the reactor, flows across the heated (and rotating at 200 
$\mathrm{rpm}$ ) susceptor and exits the reactor. Calculations were performed over the pressure range 10 - 300 Torr for $\mathrm{H}_{2}$ and for $\mathrm{N}_{2}$ gases at $13 \mathrm{slm}$. The flow simulations yield the gas heat transfer coefficient averaged over all surfaces of the susceptor. As expected this coefficient has almost no dependence on pressure.

After specifying all of the parameters discussed above, the remaining unknown is the thermal accommodation coefficient $(\alpha)$. This is in fact the value we hope to extract from the measured temperature offsets $(\Delta \mathrm{T})$ with the aid of the thermal model (assuming the structure of the model is valid). There are many ways of doing this, and we chose the following method. With all input values

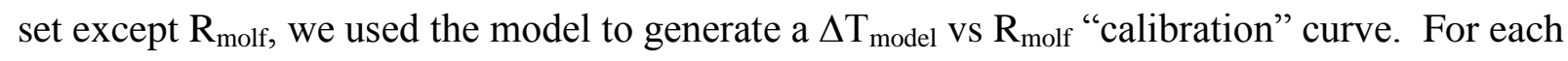
experimental $\Delta \mathrm{T}$ value we determine the corresponding $\mathrm{R}_{\text {molf }}$ value, and this in turn is plotted against the corresponding pressure. Results for $\mathrm{N}_{2}$ and $\mathrm{H}_{2}$ are shown in Fig. 4.

The log-log plot in Fig. 4 demonstrates that $\mathrm{R}_{\text {molf }}$ scales nearly perfectly as $1 / \mathrm{P}$, in accordance with eqn. 3.1. There is some departure from this scaling relationship below $\sim 20$ Torr, which will be discussed below. The fits to the data (for $\mathrm{P} \geq 20$ Torr) using a $\beta / \mathrm{P}$ function can be used to determine that $\alpha\left(\mathrm{N}_{2}\right)=0.33$ and $\alpha\left(\mathrm{H}_{2}\right)=0.13$. The $\alpha$ values are actually a composite of the thermal accommodation coefficients for the two surfaces that the gas is exchanging energy with, $\alpha_{1}$ and $\alpha_{2}$, as given by Eqn. 3.10 [ref 30, eqn. 1.137].

$$
\alpha=\frac{1}{\frac{1}{\alpha_{1}}+\frac{1}{\alpha_{2}}+1}
$$

If we assume $\alpha_{1}=\alpha_{2}$, then $\alpha_{1}\left(\mathrm{~N}_{2}\right)=0.50$ and $\alpha_{1}\left(\mathrm{H}_{2}\right)=0.23$. These values are in the range typically observed [ref 30, table 1.13], and demonstrate that on a per molecule-surface collision basis $\mathrm{H}_{2}$ is actually less efficient $(\sim 0.4 \mathrm{X})$ than $\mathrm{N}_{2}$ at exchanging energy with the two surfaces. However, since the collision rate for $\mathrm{H}_{2}$ is $\sqrt{14}$ times higher than that for $\mathrm{N}_{2}$, the net heat transfer rate for $\mathrm{H}_{2}$ is $1.5 \mathrm{X}$ higher. 
Using the measured $\alpha$ values we can now directly compare the overall model predictions of $\Delta \mathrm{T}$ as a function of pressure with the experimental observations, see Fig. 5. Note that the agreement between the model (solid) curves and experimental data (symbol) is good at pressures above 20 Torr. The disparity that arises below 20 Torr is simply another manifestation of the deviation seen in Fig. 4 at the lowest pressures. It appears that the root cause of this deviation is that as $\mathbf{R}_{\text {molf }} \rightarrow \infty(\mathrm{P} \rightarrow 0)$ the temperature offset $(\Delta \mathrm{T})$ is asymptotically approaching the value defined by $\mathbf{R}_{1 \text { rad. }}$ For the optical constants used in the model this limiting $\Delta \mathrm{T}_{\mathrm{P}=0}$ value is $\sim 480^{\circ} \mathrm{C}$, while the experimental curves appear to converge to a $\Delta \mathrm{T}_{\mathrm{P}=0} \sim 650^{\circ} \mathrm{C}$. For comparison, if we set $\tau_{2}=0$ the model extrapolates to an even lower $\Delta \mathrm{T}_{\mathrm{P}=0} \sim 350^{\circ} \mathrm{C}$, so the addition of the transmission component (with $\tau_{2}=0.5$ ) does improve model performance. Increasing the transmission value $\left(\tau_{2}\right)$ above 0.5 also tends to give better agreement (i.e. increases $\Delta \mathrm{T}_{\mathrm{P}=0}$ ), but we cannot achieve an exact fit using optical parameters we can currently justify, within the gray-body framework. In fact, we believe it is the large spectral mismatch between $\mathrm{TaC}$ and sapphire that is limiting the accuracy of the gray-body approximation. Sapphire only significantly emits (and absorbs) radiation at wavelengths longer than $\sim 4$ microns, while the emission for $\mathrm{TaC}$ is skewed towards shorter wavelengths; higher in the visible and lower in the infrared $(\varepsilon=0.6$ at $500 \mathrm{~nm}, \varepsilon=0.2$ at $4000 \mathrm{~nm}$ ). In the gray-body analysis emissivity is assumed to be constant at all wavelengths, so in material systems with large spectral differences it might not accurately describe the finer details of radiative transport. Currently, near $1300^{\circ} \mathrm{C}$ this error is only obvious below 20 Torr; at lower temperatures the error should be even smaller, but at higher temperatures it will be more of a problem. Improvements to the model will need to include some $\mathrm{TaC}$ and sapphire spectral information (go beyond the gray body approximation), and will be the subject of future work.

As a final point we examine the fraction of heat that is transferred from the susceptor to the wafer (and absorbed) via radiative transfer $\left(\boldsymbol{F} \boldsymbol{q}_{\text {Irad }}\right)$ and gas conduction $\left(\boldsymbol{F} \boldsymbol{q}_{\text {Imolf }}\right)$, where $\boldsymbol{F} \boldsymbol{q}_{\text {Imolf }}=\boldsymbol{q}_{\text {Imolf }}\left(\boldsymbol{\rho}_{\text {waf }}\right.$ $\left.\boldsymbol{q}_{\text {1trans }}\right)$ and $\boldsymbol{F} \boldsymbol{q}_{\text {lrad }}=\mathbf{1}-\boldsymbol{F} \boldsymbol{q}_{\text {Imolf. }}$. The transmission component that bypasses the wafer $\left(\boldsymbol{q}_{\text {ltrans }}\right)$ is subtracted from the total head flow since this radiation was not absorbed. Trends for $\boldsymbol{F q}_{\mathbf{q m o l f}}$ with 
temperature and pressure are shown in Table 3.1. In all cases the majority of the heat is transported to the wafer by gas conduction in the small gap between the wafer and susceptor. As the temperature is raised this fraction falls and the fraction of heat carried by radiation increases; the trend is more obvious at lower pressure where $\mathbf{R}_{\text {molf }}$ is larger. The gray-body approximation discussed earlier will limit the accuracy of these values at high temperatures and low pressures, but the trends will hold true.

\section{Summary}

We have used a MIR pyrometer to measure the absolute sapphire wafer temperature near $1300^{\circ} \mathrm{C}$, and found that it exhibits a large reactor pressure dependence. At constant RF power, when the pressure is lowered from 300 Torr to 10 Torr, the sapphire temperature drops $\sim 250^{\circ} \mathrm{C}$ while the susceptor temperature rises $\sim 25^{\circ} \mathrm{C}$. The effect is larger in $\mathrm{N}_{2}$ as compared to $\mathrm{H}_{2}$. The temperature difference between susceptor and wafer $(\Delta \mathrm{T})$ scales approximately as $1 / \mathrm{P}$, which suggests that heat conduction by the gas in the free molecular flow regime is the dominant heat transfer component. In the continuum limit gas conductivity is nearly independent of pressure, but gas filling the small gap between the wafer and susceptor transfers heat by a different mechanism, and the heat transfer rate is first order in pressure. In order to examine the issue quantitatively we built a simple "quasi-2D" model that includes the effect of sapphire transmission in the radiation exchange components. After using appropriate high temperature optical constants $(\varepsilon, \tau, \mathrm{R})$ for TaC and sapphire we have used the model to extract the heat transfer coefficient for the molecular flow component, and found that it well obeys the 1/P dependence from 20 to 300 Torr. The corresponding thermal accommodation coefficients $(\alpha)$ for $\mathrm{N}_{2}$ and $\mathrm{H}_{2}$ are measured and found to be; $\alpha_{1}\left(\mathrm{~N}_{2}\right)=0.50$ and $\alpha_{1}\left(\mathrm{H}_{2}\right)=0.23$, which are in the expected range for these values. Hydrogen, as is normally observed, is actually a less efficient transporter of energy than $\mathrm{N}_{2}$, on a per molecule-surface collision basis. But the much higher collision rate for $\mathrm{H}_{2}$ compensates for this deficiency, and the overall heat transfer rate for $\mathrm{H}_{2}$ is $\sim 1.5 \mathrm{X}$ that of $\mathrm{N}_{2}$. In general, the vast majority of heat exchanged between the susceptor and the wafer occurs by the gas molecules, 
with only a small fraction via radiation transfer. As the temperature is raised, and/or the pressure is lowered, the radiative component becomes more important, but is still $<40 \%$ for the conditions examined. A small disparity between the model and experiment arises when the pressure drops below 20 Torr, which is the region where the radiative component of heat exchange is becoming more evident. The disparity is likely due to inadequacies of the gray body approximation, given the large mismatch between the optical properties of $\mathrm{TaC}$ and sapphire. Future model improvements will need to go beyond the gray body approximation and include some spectral properties of the two materials.

Acknowledgement. This work was supported by the Laboratory Directed Research and Development program at Sandia. Sandia National Laboratories is a multi-program laboratory managed and operated by Sandia Corporation, a wholly owned subsidiary of Lockheed Martin Corporation, for the U.S. Department of Energy's National Nuclear Security Administration under contract DE-AC0494AL85000. The authors thank Prof. Zlatko Sitar of North Carolina State University for his expertise and design of the MOCVD chamber used in this work. 


\section{References:}

(1) J. Han, J.J. Figiel, M.H. Crawford, M.A. Banas, M.E. Bartram, R.M. Biefeld, Y.K. Song, A.V. Nurmikko, J. Crystal Growth 195 (1998) 291.

2) F. Nakamura, S. Hashimoto, M. Hara, S. Imanaga, M. Ikeda, H. Kawai, J. Crystal Growth 195 (1998) 280.

3) C.H.Chen, H. Liu, D. Steigerwald,W. Imler,C.P. Kuo,M.G. Craford, M. Ludowise, S. Lester, J. Amano, J. Electron. Mater. 25 (1996) 1004.

4) T.G. Mihopoulos, V. Gupta, K.F. Jensen, J. Crystal Growth 195(1998) 733.

5) J.R. Creighton, W.G. Breiland, M.E. Coltrin, R.P. Pawlowski, Appl. Phys. Lett. 81 (2002) 2626.

6) J.R. Creighton, G.T. Wang, W.G. Breiland, M.E. Coltrin, J. Crystal Growth 261 (2004) 204.

7) J.R. Creighton, J. Electron. Mater. 31 (2002) 1337.

8) G.T. Wang, J.R. Creighton, J. Phys. Chem. A. 108 (2004) 4873.

9) M.E. Coltrin, J.R. Creighton, C.C. Mitchell, J. Crystal Growth 287 (2006) 566.

10) E.V. Yakovlev, R.A. Talalaev, A.N. Vorob’ev, Electrochem. Soc. Proc. 2003-08 (2003) 258.

11) R.A. Talalaev, E.V. Yakovlev, S.Y. Karpov, I.Y. Evstratov, A.N. Vorobev, Y.N. Makarov, Phys. Status Solidi A 176 (1999) 253.

12)S. A. Safvi, J. M. Redwing, A. Thon, J. S. Flynn, M. A. Tischler and T. F. Kuech (1996) MRS Proceedings, 449, 101

13) J.R. Creighton, G.T. Wang, J. Phys. Chem. A. 109 (2005) 10554

14) J.R. Creighton, G.T. Wang, J. Phys. Chem. A. 109 (2005) 136.

15) J.R. Creighton, G.T. Wang, M.E. Coltrin, J. Crystal Growth 298 (2007) 2.

16) J.R. Creighton, D.D. Koleske, C.C. Mitchell, J. Crystal Growth 287 (2006) 572

17) Creighton, J. R.; Breiland, W. G.; Koleske, D. D.; Thaler, G.; Crawford, M. H. J. Crystal Growth $310(2008) 1062$

18) V. Hoffmann , A. Knauer, C. Brunner, S.Einfeldt, M.Weyers, G.Tränkle, K.Haberland, J.-T. Zettler, and M.Kneissl, Journal ofCrystalGrowth 315(2011)5

19) T. Bergunde, B. Henninger, M. L., M. Heuken, M. Weyersa, J.-T. Zettler, Journal of Crystal Growth 248 (2003) 235.

20) M. Belousov, B. Volf, J.C. Ramer, E.A. Armour, A. Gurary, Journal of Crystal Growth 272 (2004) 94

21) S. Mita, R. Collazo, A. Rice, R. F. Dalmau, and Z. Sitar, Journal of Applied Physics 104, 013521 (2008). 
22) R. Collazo, J. Xie, A. Rice, J. Tweedie, R. F. Dalmau, and Z. Sitar, Physica Status Solidi C 8, 2031 (2011).

23) R. Collazo, S. Mita, A. Aleksov, R. Schlesser, and Z. Sitar, Journal of Crystal Growth 287, 586-590 (2006).

24) W. G. Breiland, G. H. Evans, J. Electrochem. Soc. 138 (1991) 1806.

25) W. G. Breiland, M. E. Coltrin, J. R. Creighton, H. Hou, H. K. Moffat, J. Y. Tsao, Materials Science and Engineering, R24 (1999) 241.

26) J. R. Creighton and K. C. Baucom, Surface Science, 409 (1998) 372.

27) D. A. Gryvnak and D. E. Burce, Journal of the Optical Society of America, 55(1965)625.

28) R. P. Tye, Ed.,Thermal Conductivity, Chapter 5, Academic Press 1969.

29) C. V. Madhusudana, Thermal Contact Conductance, Chapter 4, Springer, Switzerland, 2014

30) S. Dushman, Scientific Foundations of Vacuum Technique, $2^{\text {nd }}$ Ed., John Wiley, New York, 1962.

31) Y. S. Touloukian and D. P. Dewitt, Thermophysical Properties of Matter, Volume 8, IFI/Plenum, New York, 1972.

32) D. P. Dewitt and G. D. Nutter, Eds., Theory and Practice of Radiation Thermometry, John Wiley, New York, 1988.

33) ANSYS FLUENT 170, User's Guide, ANSYS, 2016. 
Table 3.1 Fraction of heat carried to wafer by gas conduction

\begin{tabular}{|c|c|c|}
\hline Temperature $\left({ }^{\circ} \mathrm{C}\right)$ & $\boldsymbol{F q}_{\text {Imolf }}\left(20\right.$ Torr $\left.\mathrm{N}_{2}\right)$ & $\boldsymbol{F}_{\text {Imolf }}\left(200\right.$ Torr $\left.\mathrm{N}_{2}\right)$ \\
\hline 1000 & 0.87 & 0.98 \\
\hline 1200 & 0.82 & 0.97 \\
\hline 1400 & 0.75 & 0.96 \\
\hline 1600 & 0.69 & 0.95 \\
\hline
\end{tabular}




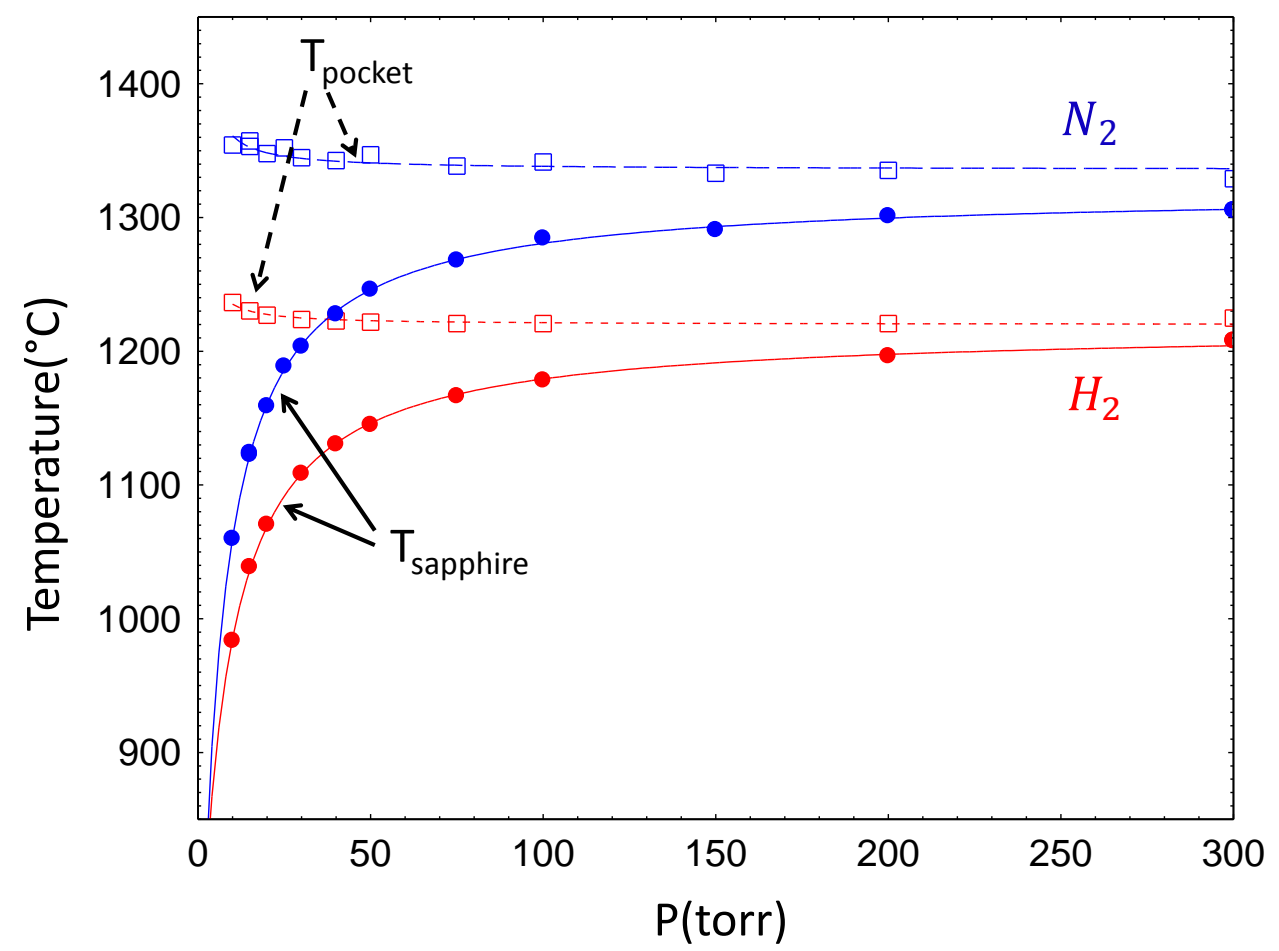

Figure 1 Temperature of as function of reactor pressure; sapphire wafer temperature $-\mathbf{s o l i d}$ circles $\left(\right.$ red $=\mathbf{H}_{2}$, blue $=$ $\mathrm{N}_{2}$ ), wafer pocket temperature - open squares $\left(\mathrm{red}=\mathrm{H}_{2}\right.$, blue $\left.=\mathrm{N}_{2}\right)$. Flow rate $=13$ slm, and $\mathrm{RF}$ power absorbed $=$ $2.5 \mathrm{~kW}$. Solid and dashed lines are empirical fits. 


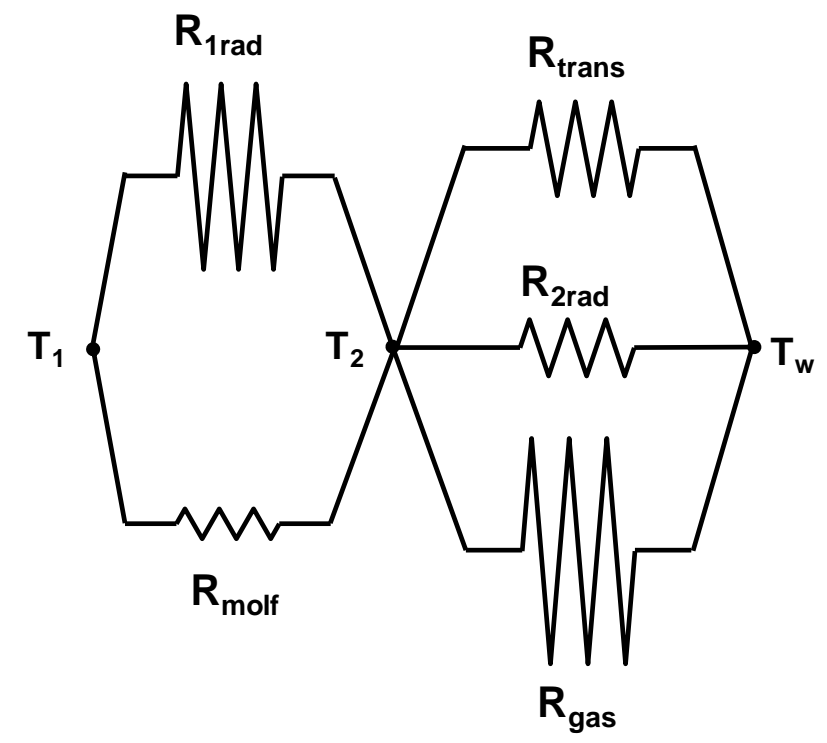

Figure 2. Equivalent thermal circuit (1D); $T_{1}=$ susceptor temperature, $T_{2}=$ wafer temperature, $T_{w}=$ wall temperature (see text for other term definitions)

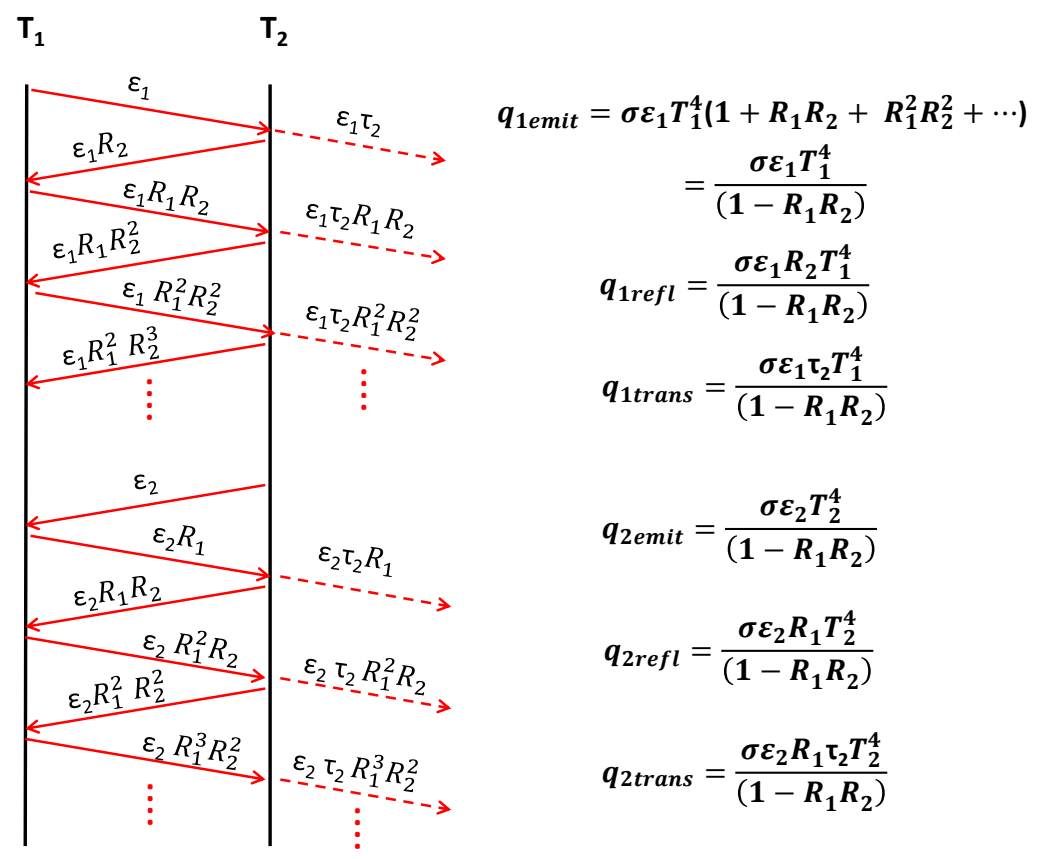

Figure 3. Derivation of the radiation components for two infinite surfaces at $T_{1}$ and $T_{2}$, with surface-1 being opaque; $R_{1}=1-\varepsilon_{1}$, and surface-2 being semi-transparent; $R_{2}=1-\varepsilon_{2}-\tau_{2}, \tau_{2}>0$. 


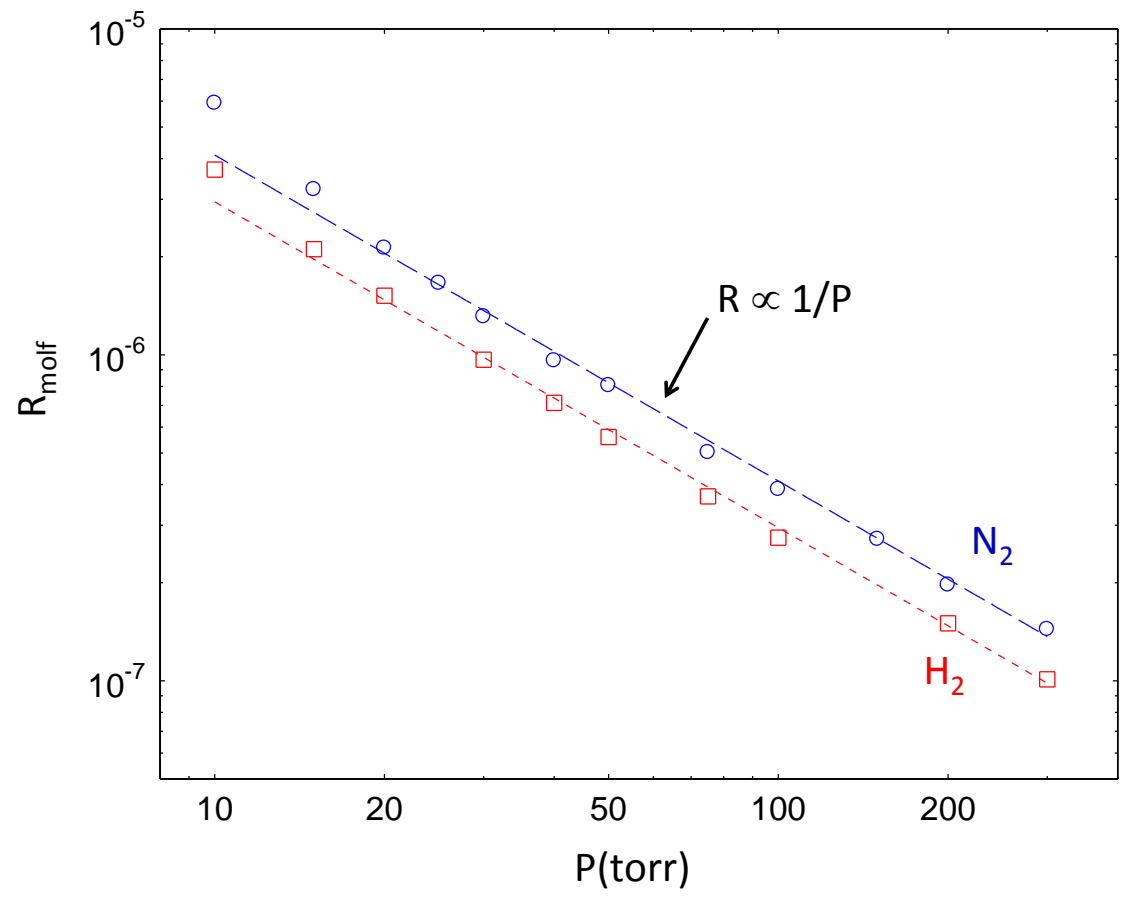

Figure 4. $\mathbf{R}_{\text {molf }}$ (deg-cm ${ }^{2}$-sec/ergs) versus Pressure for $\mathrm{N}_{2}$ (blue-circles) and $\mathrm{H}_{2}$ (red squares)

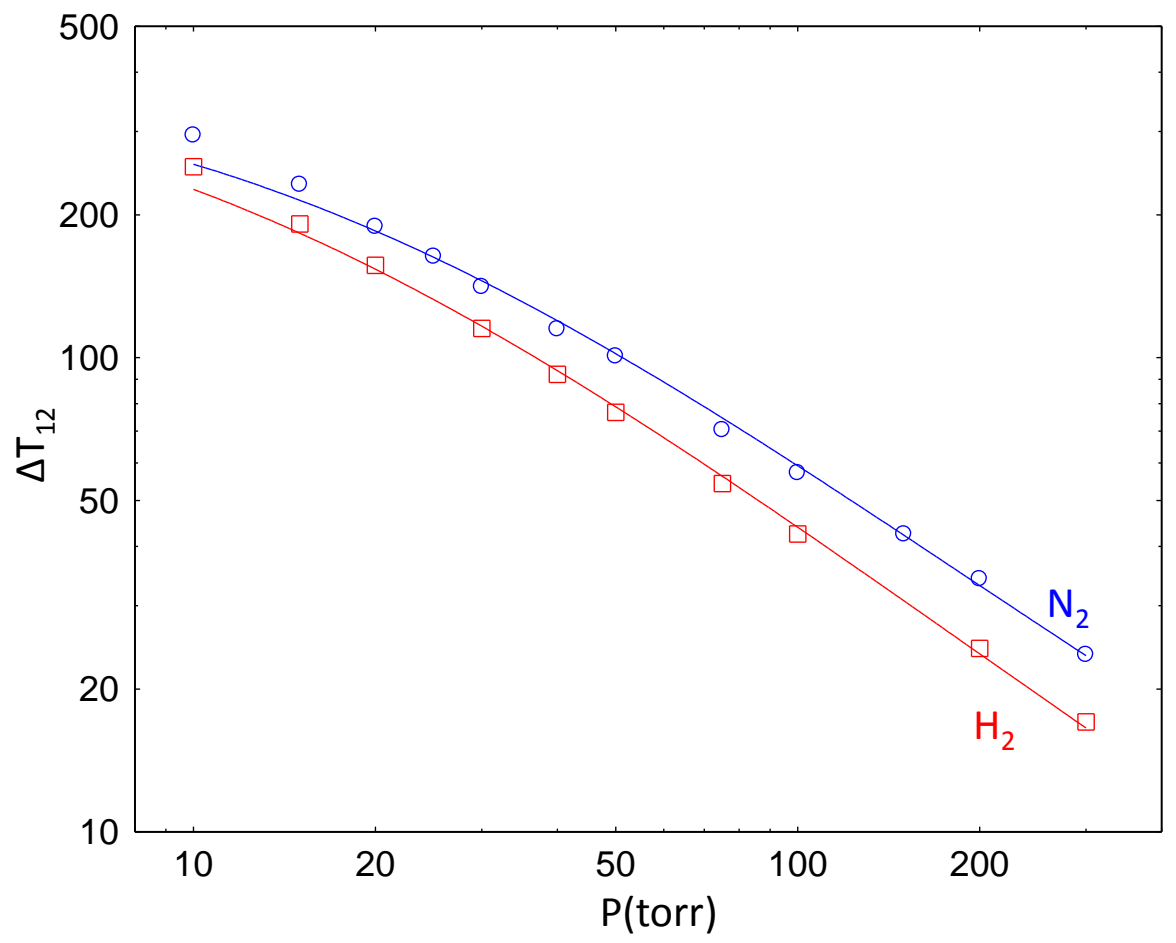

Figure 5. Comparison of model predictions (solid lines) with experimental values (symbols) 